\title{
Breast Cancer Classification Enhancement Based on Entropy Method
}

\author{
Ali Salem Ali Bin Sama ${ }^{1,2}$ \\ ${ }^{1}$ Department of Geology Engineering, Faculty of Oil \& \\ Minerals, Aden University, Yemen. \\ ${ }^{2}$ Department of Computer Science and Information, \\ College of Sharia and Islamic Studies at Al-Ahsa, Imam \\ Muhammad bin Saud Islamic University, Saudi Arabia
}

\author{
Saeed Mohammed Saeed Baneamoon \\ Department of Computer Engineering \\ Faculty of Engineering \& Petroleum \\ Hadhramout University, Yemen
}

\begin{abstract}
Breast cancer is the most common form of cancer diseases among women and the second leading cause of cancer deaths worldwide. However, due to some limitations in mammography, it is difficult to classify a suspicious mass in the breast as malignant (cancerous) or benign. This paper attempts to classify the mammographic masses with high accuracy by combining entropy method with evolutionary algorithm (EA) and fuzzy logic. EA and fuzzy logic are applied at training phase for parameters tuning, however, entropy method is applied at training phase and localization phase, where at training phase entropy enhances indicator of fuzziness, while at mass phase entropy enhances EA for the classification of masses in mammogram images. The proposed method is evaluated by experimenting a number of the benchmark Mini-MIAS databases and the results shows better and more effective identification.
\end{abstract}

Keywords-Breast Cancer, Evolutionary Algorithms, Fuzzy Logic, Entropy Method, Mammogram images and Feature Extraction)

\section{INTRODUCTION}

Breast cancer is one of the leading causes of mortality among women. It is expected that more than 26 thousand new cases among African American women to occur in US. The earliest sign of breast cancer is often an abnormality detected on mammogram screening. Early detection of breast cancer increases the possibility of recovery from the disease. Therefore, the most effective tool for detecting of breast abnormality, mammography screening have been used. Hence, for breast cancer classification, this paper presents a method by using EA, fuzzy logic and entropy method, where entropy method combine with fuzzy logic at training phase and with EA at mass phase and make the breast cancer classification process more efficient, robust and relatively fast.

\section{RELATED WORK}

A lot of approaches have been published in the literature for mammographic mass detection. A digitalized mammogram online and generates a high quality filtered segmentation of an image for biological interpretation and a texturefeature based diagnosis is needed. SVM model was regarded as an effective classifier and it showed good performances as compared with other classification models [1].

Besides that, SVM has been widely used for breast mammographic mass recognition. For example, Khan, et.al introduce and optimized Gabor filters with SVM classifier to recognizes the input ROI as malignant vs. benign[2]. The outcomes demonstrate encourage achievements of SVM against other models. The integration of SVM with PSO algorithm was discussed by Zyout etl.al [3]. In their work, hybrid of texture feature extraction techniques was implemented. By selecting the most effective texture features, the results that PSO able to reduce the complexity of the system has been indicated. Besides that, SVM was adopted for ROI mass classification in the work of Kanadam and Chereddy[4]. The main idea behind their work is to tackle the problem of mass shape variation. They introduce a new sparesROI for shape representation and the outcomes show increase in the recognition performances. The synergy of SVM with Extreme Learning Machine (ELM) was studied by Lima et.al [5]. Convolution Neural Network (CNN) was employed in their developed scheme. Mainly, their CCNA-basedscheme consists of two cascaded layers i.e. low-level feature layer and highlevel feature layer. The scheme was evaluated on a total of 600 mammographic images from DDSM database and the results indicate superior performances for the two-stage scheme over other single stage models. Additional work that integrates CNN with linear SVM was discussed by Arevalo et.al[6]. Extreme Learning Machine (ELM) based model was presented by Xie et.al [7]. In their paper ELM classifier was adopted with a set of texture features extracted from mass internal region, background region, and mass. The reported results show competitive achievements for ELM whoever inaccurate extraction of mass boundary will effect recognition performances. Besides SVM-based breast cancer diagnosis, there are a lot techniques have been developed such as Fuzzy cmean based[8], Genetic based[9], Linear Discriminate Analysis (LDA) based [10],Artificial Immune based [11],AdaBoost based[12], and level-set based [13]. 


\section{BACKGROUND}

\section{A. Evolutionary Algorithm}

Evolutionary Algorithms (EA) are stochastic search and optimization heuristics, a subset of evolutionary computation as EA for generic population-based met heuristic optimization algorithm. Evolutionary computation techniques are optimization tools derived from the classic evolution theory of biological life in the natural world such as reproduction, recombination, mutation, and selection. An optimal solution is discovered by successively breeding generations assessed by an objective "fitness" function, which they are always working on whole populations of possible solutions for a given task.

For meeting a certain selection criteria, only those individuals of a population reproduce have been the basic idea, while the population of other individuals were died, for selecting the best meet of criteria, the population of those individuals have been converged. If imperfect reproduction is added the population can begin to explore the search space and will move to individuals that have an increased selection probability and that inherit this property to their descendants. These population dynamics follow the basic rule of the Darwinistic evolution theory [14]. The general EA process is shown in Figure 1.

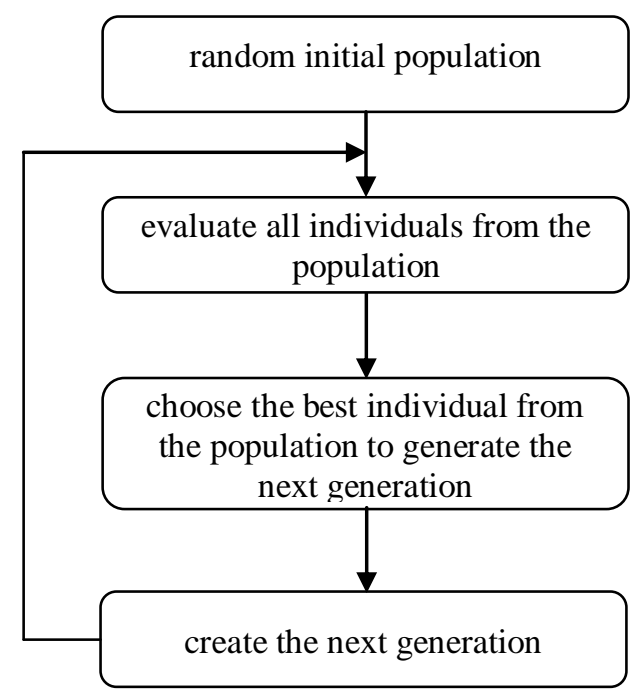

Figure 1. The general EA process

\section{B. Fuzzy Logic}

Fuzzy logic is based on fuzzy sets. In classical set theory there is a distinct difference between elements which belong to a set and those that do not. On the other hand, in the fuzzy approach, each element has a degree of membership to a set.

The main four types of shapes in Fuzzy Theory are Triangular, Trapezoidal, Gaussian (bell-shaped) and Singleton. On the other hand the general structure of Fuzzy logic system consist of four main components are fuzzification Interface, knowledge base, interface engine and defuzzification Interface as shown in Figure 2.

The fuzzy system designer must choose among several possible definitions of defuzzication. The two main methods of defuzzication: the method of the mean of maxima (MeOM) and the method of center of gravity (COG), where the COG defuzzication is more commonly used [15].

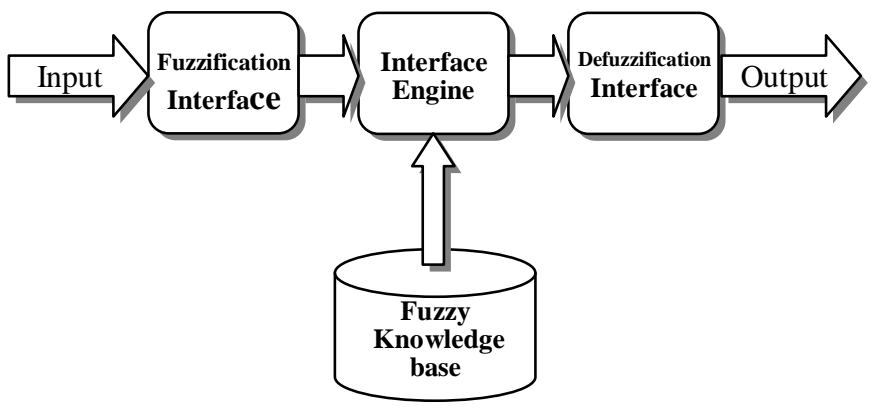

Figure 2. Overview diagram of a fuzzy system

\section{Entropy Method}

In information theory, Entropy is defined as the measure of uncertainty associated with a random variable. The term usually refers to the Shannon entropy, which quantifies - in the sense of an expected value. In other words, entropy is a measure of unpredictability of the state, or equivalently, of its average information content.

The Shannon entropy $H(X)$ of a random variable $X$ with probability mass function $\mathrm{p}(\mathrm{x})$ can be used to measure the average information content that is missing when the value of $\mathrm{X}$ is unknown [16].

Shannon defined the entropy for discrete variables as:

$$
H(X)=\sum_{i=1}^{m} p_{i} \cdot \log _{2} \frac{1}{p_{i}}=-\sum_{i=1}^{m} p_{i} \cdot \log _{2} p_{i}
$$

where for given events $e_{1}, \ldots ., e_{m}$ occurring with probabilities $p_{1}, \ldots ., p_{m}, p_{i}$ is equal to the chance of observing value $i$ and $\log _{2}$ is the logarithm with base 2 .

Shannon's entropy for an image can be computed such as a probabilistic method for comparing two pixels or a group of pixels and the probabilities of the gray level distributions. In evolutionary algorithm, entropy can disclose important information on EA parameters, where entropy can be used to indicate how influential a particular parameter is on EA performance. In Fuzzy logic, entropy method is used to conduct goal-driven calculations by processing the data that have been transformed into a fuzzy structure. Therefore, Fuzzy entropy is used to express the mathematical values of the fuzziness of fuzzy sets. 


\section{PROPOSED METHOD}

The paper presents a method for enhancement breast censer classification by combining entropy method with evolutionary algorithm and fuzzy logic. Entropy method is applied at training phase and localization phase, where at training phase entropy enhances indicator of fuzziness, while at mass phase entropy enhances EA for the classification of masses in mammogram images.

The proposed method consists of three stages as shown in Figure 3.The details are as follows:

Stage 1: The radiology marks the unknown mass with a square window as indicated in Figure 3.

Stage 2: Entropy method with evolutionary algorithm will be used to extract the features from the input window then a feature vector will be formed and presented to fuzzy logic classifier.

Stage 3: The trained entropy enhances indicator of fuzziness will recognize the input pattern as Benign (normal) or Malignant (abnormal) mass.

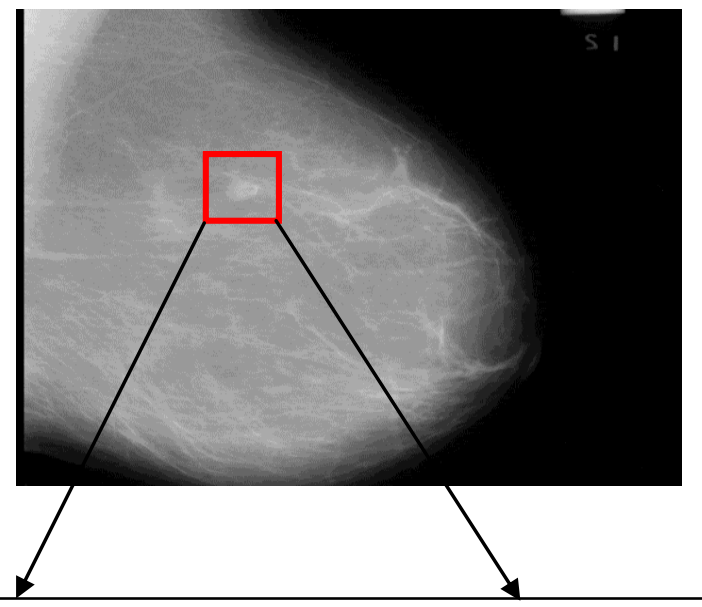

Entropy method with evolutionary algorithm

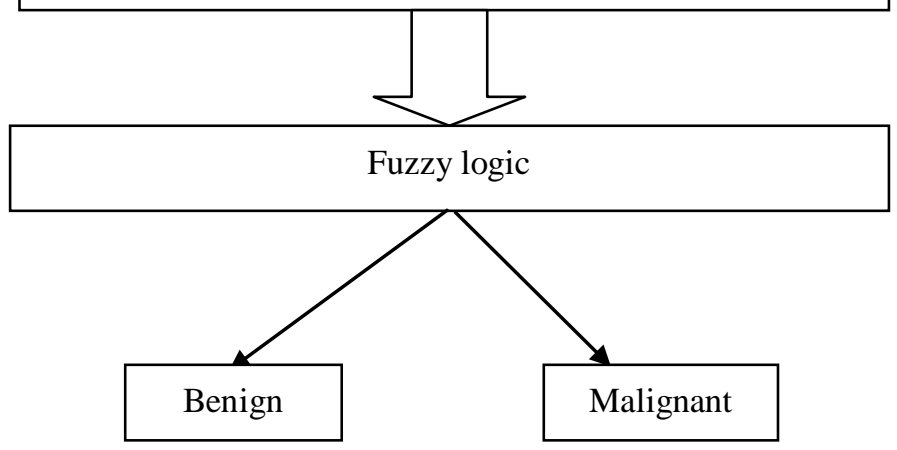

Figure 3.General View of Research Methodology

\section{EXPERIMENTAL RESULT}

A total of 500 DDSM images with 250 malignant ROIs and 250 benign ROIs have been used in this study. Moreover, a total of 1000 normal ROIs are employed. These normal ROIs have been generated randomly from various places inside the studied mammographic images. For evaluation purpose, each experiment was repeated ten times and the dataset was randomly split into three set i.e.40\% training set, $40 \%$ validation set, and $20 \%$ testing set respectively. The experimental analysis was conducted as follows.

\section{A. Entropy method for training phase}

The effects of the entropy method with respect to system performance, complexity, computational time were investigated. In the experimental study, the entropy method with fuzziness were used. Three performance indicators were computed, i.e. model complexity, computational time, and model accuracy. Each experiment was repeated ten times. The average and standard deviation of the performance indicators were computed.

Table 1: Analysis the effect of the number of classifiers on system perfomances

\begin{tabular}{|c|c|c|c|c|c|c|}
\hline \multirow[b]{2}{*}{ System } & \multicolumn{2}{|c|}{ Accuracy (\%) } & \multicolumn{2}{|c|}{ Computational time (sec) } & \multicolumn{2}{|c|}{ Complexity } \\
\hline & $\begin{array}{l}\text { Sensitivity } \\
\text { (std.dev.) }\end{array}$ & $\begin{array}{r}\text { Specificity } \\
\text { (std.dev.) }\end{array}$ & $\begin{array}{c}\text { Feature Extraction } \\
\text { (std.dev.) }\end{array}$ & $\begin{array}{c}\text { Recognition } \\
\text { (std.dev.) }\end{array}$ & $\begin{array}{l}\text { Total SVs } \\
\text { (std.dev.) }\end{array}$ & $\begin{array}{c}\text { Total Feat } \\
\text { (std.dev) }\end{array}$ \\
\hline 1 classifier & $\begin{array}{l}84.75 \\
(6.11)\end{array}$ & $\begin{array}{c}30.93 \\
(11.76)\end{array}$ & $\begin{array}{l}1.84 \\
(0.18)\end{array}$ & $\begin{array}{c}0.03 \\
(0.01)\end{array}$ & $\begin{array}{c}65.0 \\
(15.52)\end{array}$ & $\begin{array}{l}210 \\
(0)\end{array}$ \\
\hline 2 classifiers & $\begin{array}{l}77.40 \\
(4.89)\end{array}$ & $\begin{array}{l}44.10 \\
(5.09)\end{array}$ & $\begin{array}{c}1.93 \\
(0.16)\end{array}$ & $\begin{array}{c}0.05 \\
(0.01)\end{array}$ & $\begin{array}{l}118.67 \\
(9.71)\end{array}$ & $\begin{array}{l}210 \\
(0)\end{array}$ \\
\hline 3 clapsifiers & $\begin{array}{l}72.88 \\
(3.56)\end{array}$ & $\begin{array}{c}64 \\
(2.39)\end{array}$ & $\begin{array}{c}1.95 \\
(0.46)\end{array}$ & $\begin{array}{c}0.06 \\
(0.01)\end{array}$ & $\begin{array}{l}201.40 \\
(14.33)\end{array}$ & $\begin{array}{l}210 \\
(0)\end{array}$ \\
\hline
\end{tabular}

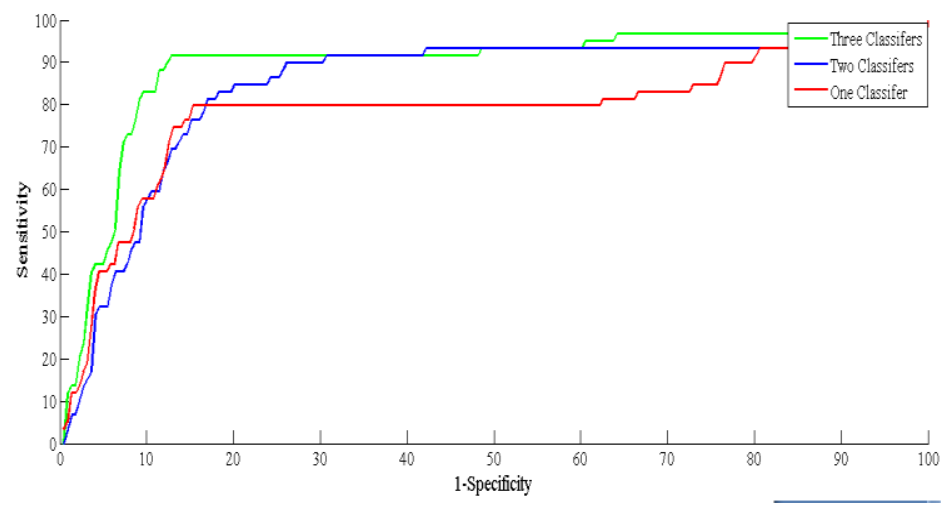

Figure 4: ROC curve results 


\section{B. Effects of the numbers of training features}

The effects of the numbers of training features $(F)$ and negative training instances $(\mathrm{N})$ were investigated. In this section, the Centre Composite Design(CCD) technique was utilized to analysis the performances at three levels i.e. low, middle, and high. Accordingly, the $\mathrm{F}$ and $\mathrm{N}$ values were set at three different levels, i.e. low, medium, and high, as shown in Table 2.

Table 2: Parameters and levels of the CCD experiment

\begin{tabular}{lccc}
\hline Parameter $\backslash$ Level & Low & Medium & High \\
\hline Number of features and negative Instances $(F, N)$ & $(5,10)$ & $(10,20)$ & $(20,40)$
\end{tabular}

Each experiment was repeated ten times. The mean and standard deviation of the numbers of features and SVs, feature extraction time, recognition time, sensitivity rate, and specificity rate were computed, as reported in Table 3 . The minimum model complexity in terms of the total number of features was achieved in Experiment 1. This was because the number of features and negative training instances were set to 5 and 10, respectively. However, when the number of features increased from Low to High, the feature extractions time increased. In addition, the recognition and specificity rate were improved by increasing the total number of features and negative training instances from Low to High, as shown in Table 3. These improvements resulted from the ability of the developed model to identify the true target patterns with higher numbers of features and negative instances.

\begin{tabular}{lccc}
\multicolumn{4}{c}{ Table 3: Experimental results of parameter analysis } \\
\hline & $\begin{array}{c}\text { Experiment } \\
\text { (Low ) }\end{array}$ & $\begin{array}{c}\text { Experiment 2 } \\
\text { (Medium) }\end{array}$ & $\begin{array}{c}\text { Experiment 3 } \\
\text { (High ) }\end{array}$ \\
\hline Total Features & 210.00 & 420.00 & 840.00 \\
Total SVs & 201.40 & 246.5 & 299.50 \\
$\begin{array}{l}\text { Feature Extraction } \\
\text { (sec) }\end{array}$ & 2.20 & 3.01 & 5.67 \\
$\begin{array}{l}\text { Recognition Time (sec) } \\
\text { Sensitivity (\%) }\end{array}$ & 0.06 & 0.09 & 0.15 \\
& & & 51.67 \\
Specificity (\%) & 72.88 & 62.97 & 78.07 \\
& 64.00 & 70.00 & \\
\hline
\end{tabular}

For graphical illustration, system complexity, computational time, and system accuracy are shown in Figure 5 It can be observed; that Experiment 1 produces the best sensitivity rate with minimum computational time of 0.06 seconds recognition time, as well as the lowest degree of complexity with SVs of 204.40 and total features of 210.

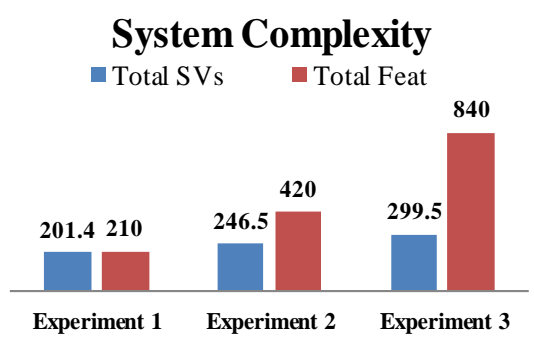

(a)

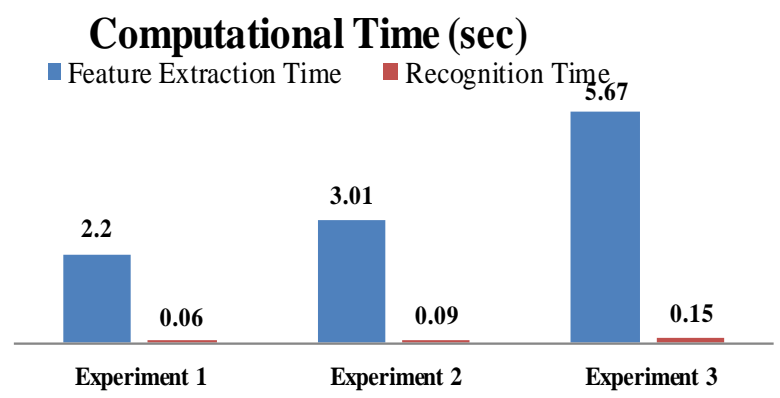

(b)

\section{System Accuracy}

- Sensitivity

Specificity

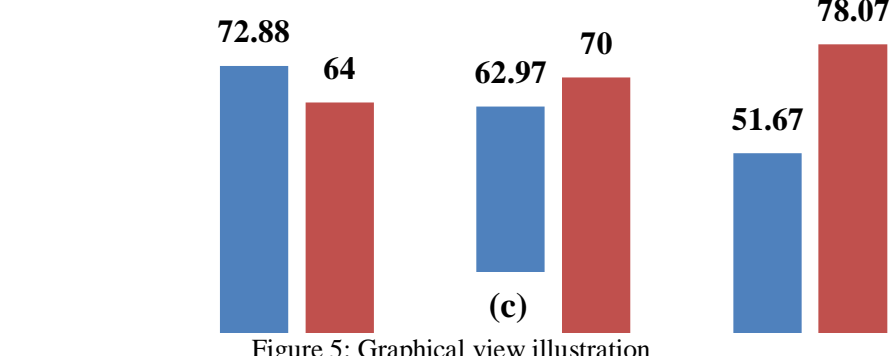

78.07

(a) System complexity (b) Computational time, and (d) System accuracy.

The Graphical User Interface (GUI) of the developed system is shown in Figures 6. To execute the system, two main steps are required i.e. ROI region labeling, and classifying the labeled region as Benign or Malignant as indicated in Figure 6

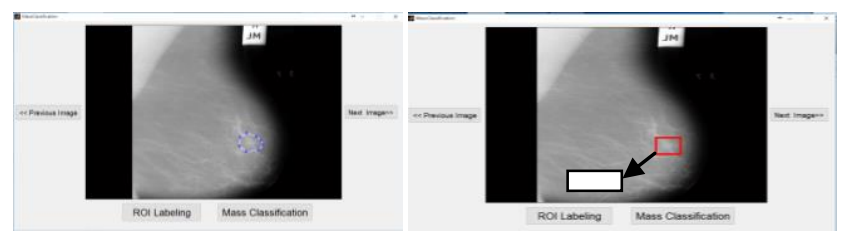

(b).

(a)

(b)

Figure 6: GUI of the enhanced breast diagnosis system (a)-(b) 


\section{CONCLUSION}

This study encompasses the effect of entropy method for classifying the masses presented at mammographic images, which depend on Shannon's entropy concept. The developed system has been evaluated with a total of 500 DDSM mammographic images (250 malignant cases, and 250 benign cases). The reported results positively show a superior performance of the presented system over other variant methods. Additionally, from the statistical point of view, the proposed system was able to achieve significant results as compared with other models. Further work could be conducted by adopting the proposed model for different breast cancer images such as MRI and ultrasound.

\section{REFERENCES}

[1] C.-W. Hsu, C.-J. Lin, A comparison of methods for multiclass support vector machines, Neural Networks, IEEE Transactions on, 13 415-425, 2002.

[2] S. Khan, M. Hussain, H. Aboalsamh, H. Mathkour, G. Bebis, M. Zakariah, Optimized Gabor features for mass classification in mammography, Applied Soft Computing, 44 , 267-280, 2016.

[3] I. Zyout, J. Czajkowska, M. Grzegorzek, Multi-scale textural feature extraction and particle swarm optimization based model selection for false positive reduction in mammography, Computerized Medical Imaging and Graphics, 46, 95-107, 2015.

[4] K.P. Kanadam, S.R. Chereddy, Mammogram classification using sparseROI: A novel representation to arbitrary shaped masses, Expert Systems with Applications, 57, 204-213, 2016.

[5] S.M. de Lima, A.G. da Silva-Filho, W.P. dos Santos, Detection and classification of masses in mammographic images in a multi-kernel approach, Computer Methods and Programs in Biomedicine, 2016.

[6] J. Arevalo, F.A. González, R. Ramos-Pollán, J.L. Oliveira, M.A.G. Lopez, Representation learning for mammography mass lesion classification with convolutional neural networks, Computer methods and programs in biomedicine, 127, 248-257, 2016.

[7] W. Xie, Y. Li, Y. Ma, Breast mass classification in digital mammography based on extreme learning machine, Neurocomputing, 173, 930-941, 2016.

[8] K. Vaidehi, T. Subashini, Automatic Characterization of Benign and Malignant Masses in Mammography, Procedia Computer Science, 46, 1762-1769, 2015.

[9] W.B. de Sampaio, A.C. Silva, A.C. de Paiva, M. Gattass, Detection of masses in mammograms with adaption to breast density using genetic algorithm, phylogenetic trees, LBP and SVM, Expert Systems with Applications, 42, 8911-8928, 2015.

[10] Y. Li, H. Chen, X. Wei, Y. Peng, L. Cheng, Mass classification in mammograms based on two-concentric masks and discriminating texton, Pattern Recognition, 60, 648-656, 2016.

[11] G. Magna, P. Casti, S.V. Jayaraman, M. Salmeri, A. Mencattini, E. Martinelli, C. Di Natale, Identification of mammography anomalies for breast cancer detection by an ensemble of classification models based on artificial immune system, Knowledge-Based Systems, 101, 60-70, 2016.

[12] F. Pak, H.R. Kanan, A. Alikhassi, Breast cancer detection and classification in digital mammography based on Non-Subsampled Contourlet Transform (NSCT) and Super Resolution, Computer methods and programs in biomedicine, 122, 89-107, 2015.
[13] R. Rouhi, M. Jafari, Classification of benign and malignant breast tumors based on hybrid level set segmentation, Expert Systems with Applications, 46, 45-59, 2016.

[14] D. Simon, Evolutionary Optimization Algorithms, Wiley, 2013.

[15] G. J. Klir and B. J. Yaun, Fuzzy Sets and Fuzzy Logic: Theory and Application, Prentice Hall of India, 1997.

[16] C.E.Shannon, A mathematical theory of communication. Bell System Technical Journal, Vol 27, pp. 379-423, 623-656, 1948.

\section{AUTHORS PROFILE}

Dr. Ali Salem Ali Bin Sama received the B.E degree in Computer Engineering, Balqa University, Faculty of Applied Engineering, Jordan, in 1997, the M.Sc. degree in Computer Science Intelligence from Universiti Sains Malaysia, USM, Penang, Malaysia, in 2006, and the $\mathrm{PhD}$ degree in Image Processing and Artificial Intelligence from Universiti Sains Malaysia, USM, Penang, Malaysia in 2011. He has published a number of papers in journals and conferences, $\mathrm{He}$ is assistant professor in department of engineering geology, Minerals \& Oil Faculty, Aden University, Yemen, Now, Assistant Professor in a Department of Computer Science and Information, College of Sharia and Islamic Studies at Al-Ahsa, Imam Muhammad bin Saud Islamic University, in Saudi Arabia. His interest focuses on the field of Artificial Intelligence and Image Processing.

Dr. Saeed Mohammed Baneamoon received the B.Sc. and the M.Sc. degrees in computer engineering from Technical University, Sofia, Bulgaria in 1996, the M.Sc. degree in computer science from University of Technology, Baghdad, Iraq in 2003 and the Ph.D. degree in Artificial Intelligence from Universiti Sains Malaysia, USM, Penang, Malaysia in 2011. He is Assistant Professor in Department of Computer Engineering, Faculty of Engineering \& Petroleum, Hadhramout University, Hadhramout, Yemen From 2011 till now. He is a Head of Depart Hadhramout, Yemen From 2014 till now. His interest focuses on the field of Artificial Intelligence and Robotics. He has published a number of papers in journals and conferences.

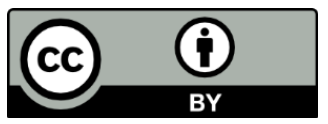

(C) 2017 by the author(s); licensee Empirical Research Press Ltd. United Kingdom. This is an open access article distributed under the terms and conditions of the Creative Commons by Attribution (CC-BY) license. (http://creativecommons.org/licenses/by/4.0/). 